\title{
Simulation of Rayleigh Bubble Growth near a No-Slip Rigid Wall
}

\author{
Tomoya Tanaka ${ }^{1, a}$ and Keita Ando ${ }^{1, b}$
}

\author{
${ }^{1}$ Keio University, 3-14-1 Hiyoshi, Kohoku-ku, Yokohama, Kanagawa 223-8622, Japan

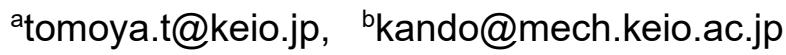

Keywords: Ultrasonic cavitation cleaning, Cavitation inception, Bubble growth, Wall shear stress

\begin{abstract}
In order to study the role of growing cavitation bubbles in the context of ultrasonic cleaning, we perform two-dimensional, axisymmetric Navier-Stokes simulation for compressible, multicomponent flow and examine the so-called Rayleigh growth of an air bubble (with initial radius $33 \mu \mathrm{m}$ and pressure $10 \mathrm{MPa}$ ) near a rigid wall. The simulation suggests that strong shear stress, which is important in physical cleaning such as particle removal, appears as a result of the bubble-growthinduced shock passage. The parametric study with varying a standoff distance of the bubble to the wall shows that the wall shear stress linearly decreases against the standoff distance.
\end{abstract}

\section{Introduction}

Ultrasonic cleaning is an essential process in semiconductor industry. Earlier studies [1,2] suggested that wall shear stress induced by dynamics of acoustic cavitation bubbles is a main factor to produce cleaning effects of underwater ultrasound. When it comes to studying acoustic cavitation bubbles near rigid boundaries, a main focus has been put on their collapse dynamics that can lead to strong wall shear stress [3,4]. Even though cavitation is expected to be nucleated heterogeneously from micron-sized or even smaller gas bubble nuclei pre-existing in the liquid bulk or at solid boundaries, wall shear stress generation from cavitation bubble nucleation (often called cavitation inception) has not been studied in the context of ultrasonic cleaning, to the author's knowledge. In this study, we aim to quantify wall shear stress as a result of the initial growth of a nucleated cavitation bubble. To do so, we perform two-dimensional, axisymmetric Navier-Stokes simulation for compressible, multicomponent flow, which allows for reproducing the bubble-growth-induced shock wave propagation and wall shear stress generation.

\section{Physical and Numerical Modeling}

The computational setup is depicted in Fig. 1. Since the scale separation between the size of cavitation bubble nuclei and the wavelength of ultrasound is very large, it is computationally expensive to fully resolve such bubble-ultrasound interaction. Here, for simplicity, we model the initial growth of a nucleated cavitation bubble as the so-called Rayleigh bubble growth [5]. The bubble growth is driven by pressure imbalance between the bubble and its ambient liquid. Rayleigh advocated the bubble collapse theory in the case of that when homogeneous fluid is at rest and spherical portion of the fluid is suddenly annihilated, the pressure at any point of the mass instantly alters, the pressure at an infinite distance being supposed to remain constant. We adapted and examined his proposed assumption to bubble growth rather than bubble collapse [6]. The bubble is assumed to consist of air that obeys the perfect gas law; the liquid is set initially at the standard temperature and pressure $\left(\mathrm{T}=20^{\circ} \mathrm{C}, p_{\infty}=101.3 \mathrm{kPa}\right)$. The initial pressure of the nucleated bubble is set at $p_{0}=10 \mathrm{MPa}$ and its density and temperature are determined according to the adiabatic relation with specific ratio $\gamma=1.4$. The initial radius of the nucleated bubble (at time $t=0$ ) is set, as an example, at $R_{0}=33 \mu \mathrm{m}$, which is expected to show large-amplitude bubble oscillation for the case of $O(10 \mathrm{kHz})$ ultrasound, and used to make length scales dimensionless. The standoff distance from the bubble center to the no-slip rigid wall (aligned with the $r$ axis at $z=0$ ) is treated as a parameter and set at $z / R_{0}=L / R_{0}=1.25,1.5,1.75$ and 2 . 


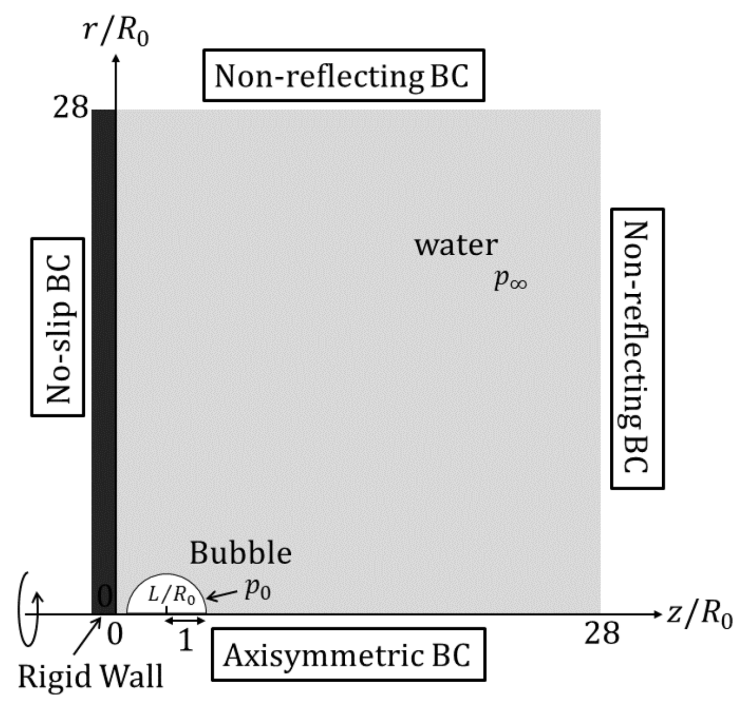

Figure 1: Problem setup of the bubble growth near a rigid wall, together with the computational domain and boundary conditions. The problem is axisymmetric with respect to $z$ axis. The bubble growth is induced by initial pressure imbalance $p_{0}>p_{\infty}$ where $p_{\infty}$ stands for one atmosphere in the liquid.

To numerically replicate the bubble-growth-induced shock propagation and shear stress generation, we solve two-dimensional, axisymmetric Navier-Stokes equations for compressible flow consisting of gas and liquid components (with no phase change). To be simple, we exclude terms related with heat conduction and surface tension from the equations, for the heat transfer is gradual in comparison to acoustic phenomena and Laplace pressure is much smaller than the pressure imbalance we consider in this study.

The numerical method we use is based on the solution-adaptive, shock-interface capturing method[7]. For spatial discretization, a third-order finite-volume weighted essentially nonoscillatory (WENO) scheme with the Harten Laxvan Leer (HLL) approximate Riemann solver is adopted only in the vicinity of discontinuities including stocks and interfaces[8]. Otherwise, the fourth-order central differencing scheme is adopted. Time integration is handled by a third-order, total variation diminishing (TVD) Runge-Kutta scheme. The calculation domain is set to be so large that the bubble is not affected by acoustic waves numerically reflected from its boundaries. No-slip, axisymmetric[9], and non-reflecting[10] boundary conditions are applied at the edges of the computational domain as explained in Fig. 1. We apply grid stretching with which the simulation is performed with cheaper cost but the flow near the bubble is resolved with finest grid spacing of $\Delta r / R_{0}=\Delta z / R_{0}=$ $3.5 \times 10^{-3}$.

\section{Results and Discussion}

As a representative example, we present the evolution of the velocity and pressure fields for the case of standoff distance $L / R_{0}=1.25$ in Fig. 2, showing the bubble-growth-induced shock propagation/reflection and subsequent liquid flow. Spatial evolution of the wall shear stress at representative times is presented in Fig. 3(a). We can see the acoustic event and its induced fluid flow: a spherical shock (as a result of the pressure imbalance between the bubble and its ambient) is initially emitted and subsequently reflected at the wall and the bubble interface. The fluid motion is induced after the shock passage and larger velocity appears in the vicinity of the growing bubble. The maximum wall shear stress $(12.3 \mathrm{kPa})$ appears at $t=20 \mathrm{~ns}$ and may be strong enough, for example, 
(a-i)

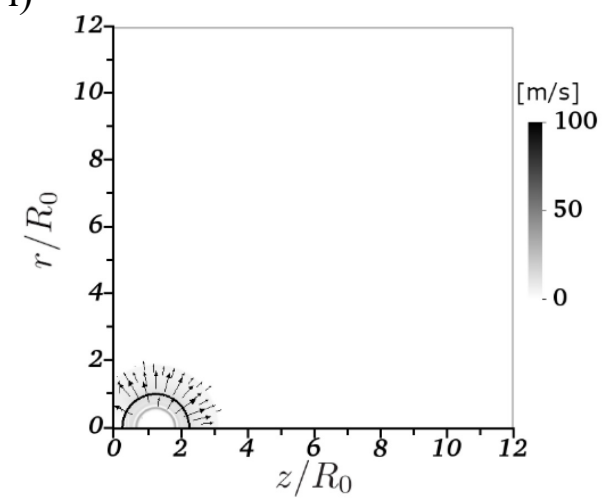

(a-ii)

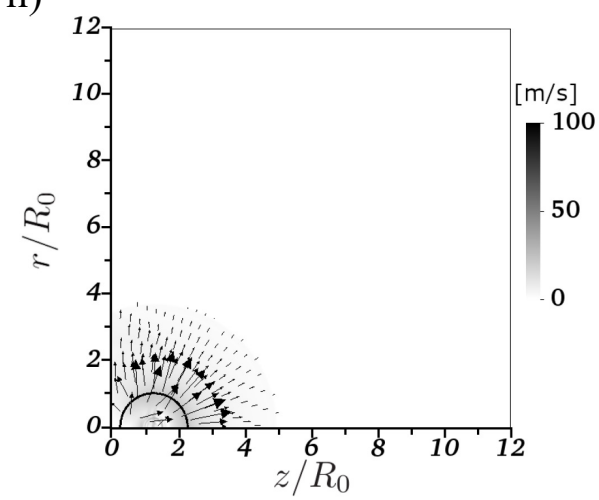

(a-iii)

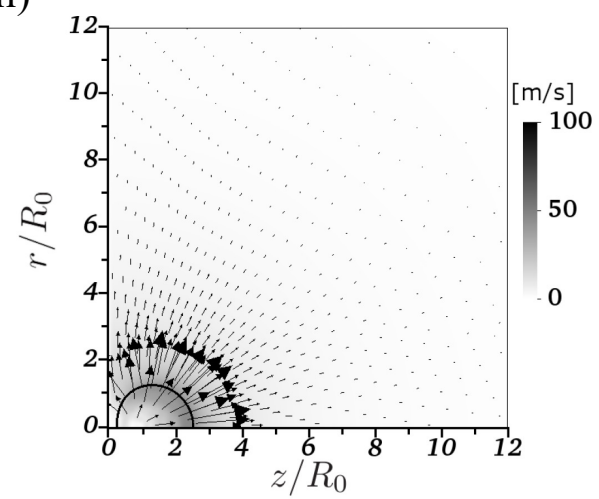

(a-iv)

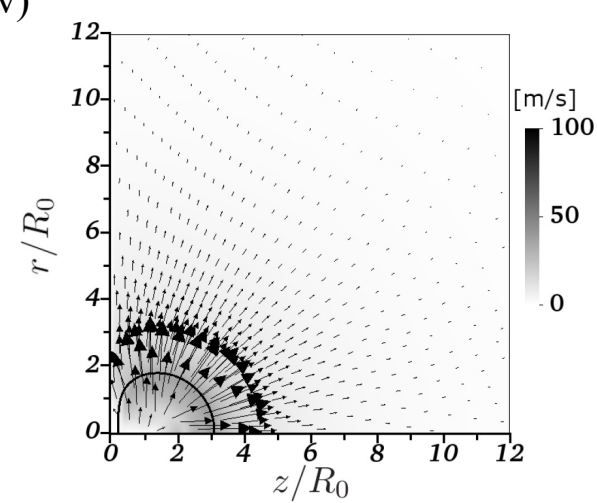

(b-i)

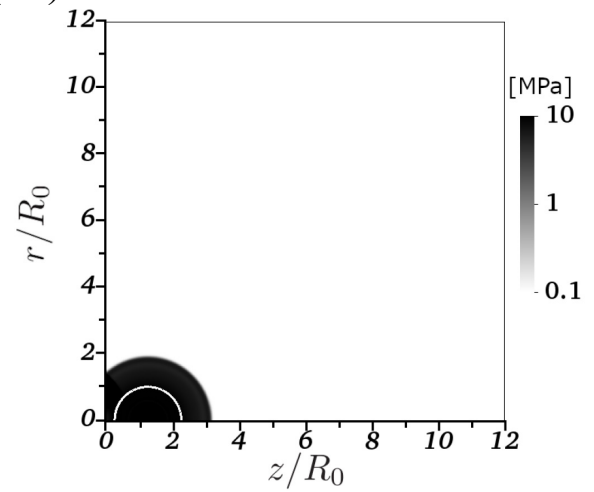

(b-ii)

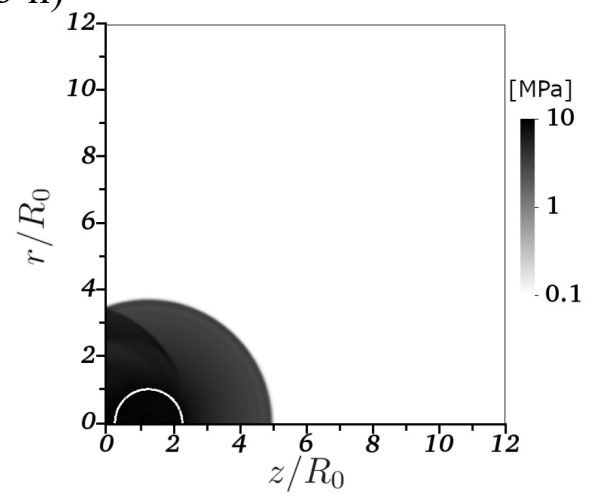

(b-iii)

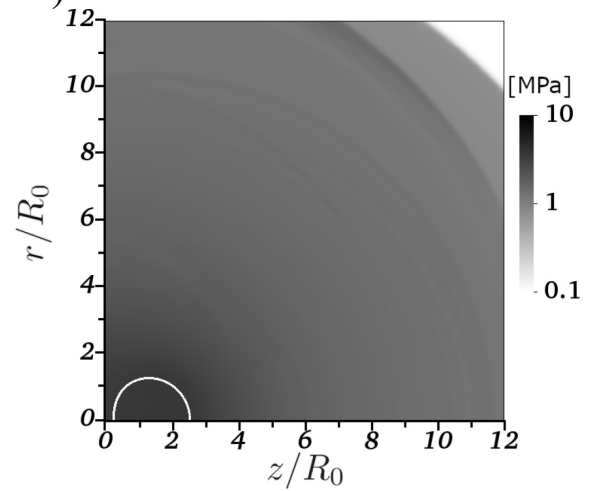

(b-iv)

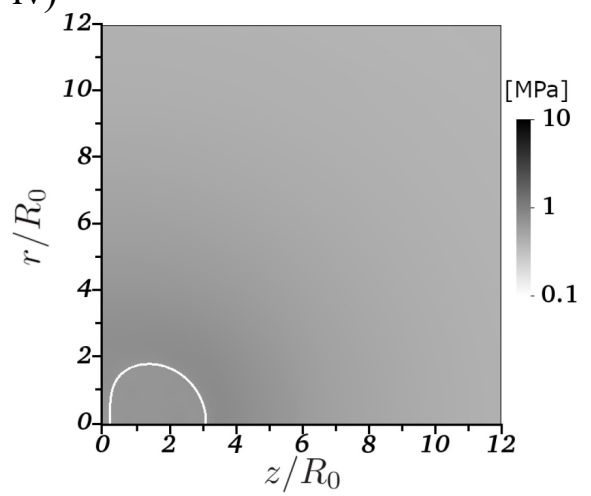

Figure 2: Snapshots of the bubble growth near the rigid wall at representative times for the case of $L / R_{0}=1.25$ : (i) $t=20 \mathrm{ns,} \mathrm{(ii)} t=60 \mathrm{~ns}$, (iii) $t=300 \mathrm{ns,} \mathrm{(iv)} t=800 \mathrm{~ns}$, plotting the distributions of (a) the velocity vectors and magnitudes $[\mathrm{m} / \mathrm{s}]$ and (b) the pressure [MPa]. Black and white lines represent the bubble interface in (a) and (b), respectively. 
to remove spherical polystyrene particles of diameter $d=10 \mathrm{~nm}$ from a quartz surface[11,12]. In Fig. $3(b)$, spatial evolutions of the wall shear stress and pressure when the maximum wall shear stress is achieved $(t=20 \mathrm{~ns})$ are shown, suggesting that the strong wall shear stress results from the shock passage with its induced flow along the wall surface.

We repeated the simulation, but with varying the standoff distance of the bubble to the wall $\left(L / R_{0}\right)$. In Fig. 4, we examine the effect of the standoff distance on the maximum wall shear stress and its generation location. It follows that the maximum wall shear stress decreases as $-6.49 L / R_{0}$ and its generation position gets larger as $0.702 L / R_{0}$, in the range of $L / R_{0}$ of our concern. We may say that the growth of bubbles nucleated closer to cleaning targets will contribute to better cleaning performance.

(a)

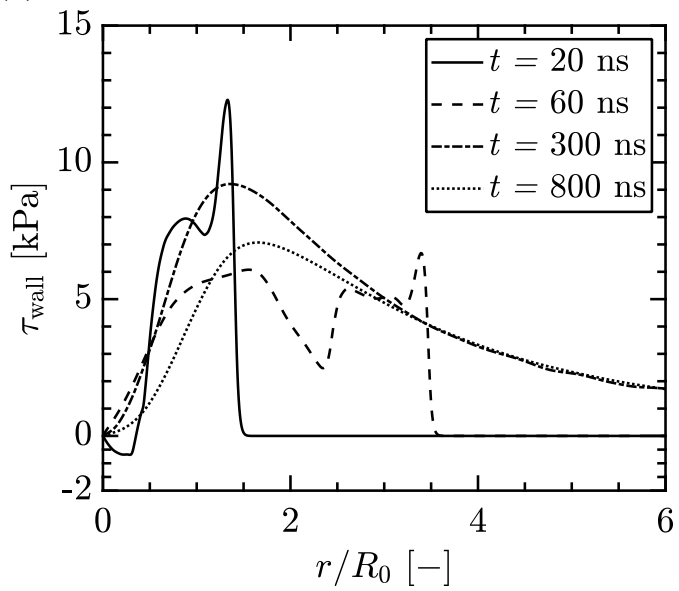

(b)

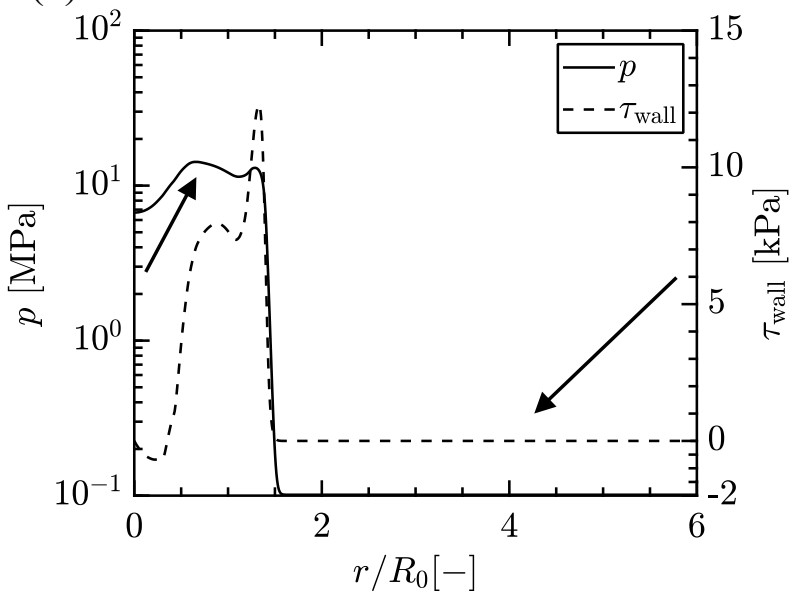

Figure 3: (a) Spatial evolution of the wall shear stress along the rigid wall $(z=0)$ at representative times for the case of $L / R_{0}=1.25$; (b) Comparison between the wall shear stress and the pressure along the rigid wall $(z=0)$ at $t=20$ ns for the case of $L / R_{0}=1.25$.

(a)

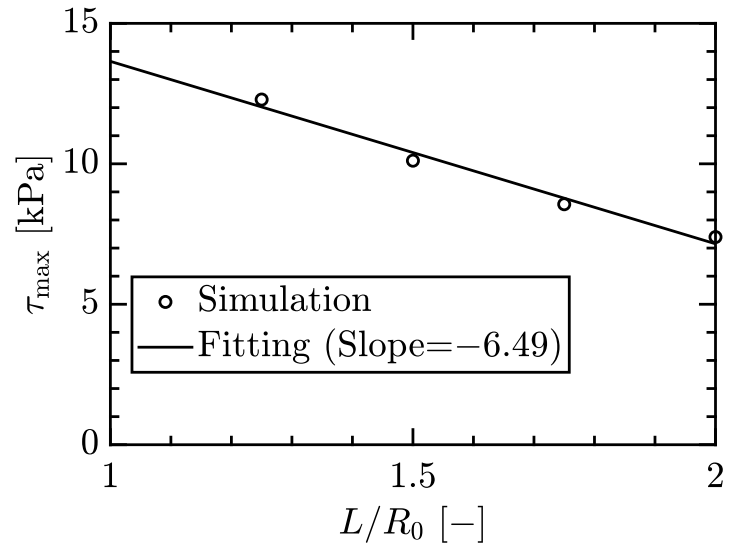

(b)

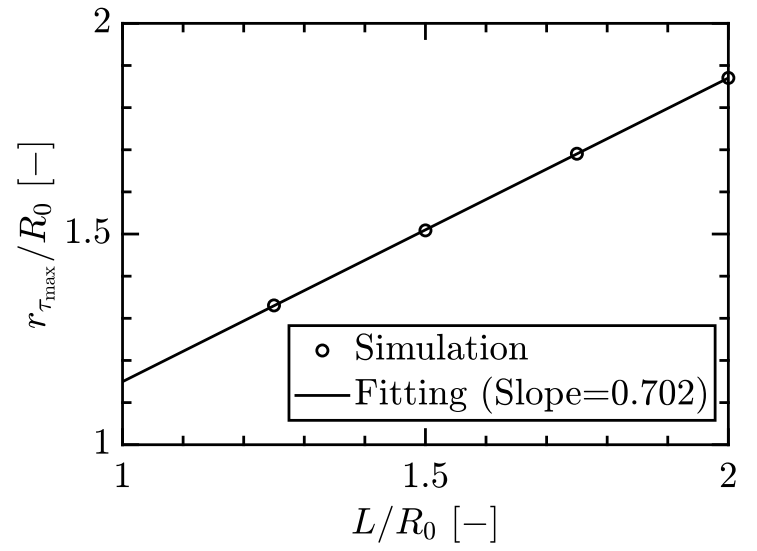

Figure 4: (a) Magnitude $\tau_{\max }$ and (b) position $r_{\tau_{\max }} / R_{0}$ of the maximum wall shear stress, as a function of the standoff distance $L / R_{0}$. The simulation data are fitted to a straight line. 


\section{Conclusion}

In short, the two-dimensional, axisymmetric compressible Navier-Stokes simulation shows strong wall shear stress generation as a result of the Rayleigh bubble growth, suggesting that cavitation bubble nucleation can play an important role in acoustic-cavitation-based cleaning. The parametric study on the standoff distance of nucleated cavitation bubbles to the no-slip wall suggests that bubbles nucleated closer to cleaning targets will play a more important role as cleaning agents.

\section{References}

[1] W. Kim et al.: Appl. Phys. Lett. 94 (2009), p. 151-153.

[2] T. Yamashita and K. Ando: Ultrason. Sonochem. 52 (2019), p. 268-279.

[3] Q. Zeng et al.: J. Fluid Mech. 846 (2018), p. 341-355.

[4] P. Koukouvinis et al:: Langmuir 34 (2018), p. 6428-6442.

[5] C. E. Brennen: Cavitation and Bubble Dynamics (Cambridge University Press, UK, 2013).

[6] L. Rayleigh: Phil. Mag. 34 (1917), p. 94-98.

[7] S. A. Beig and E. Johnsen: J. Comput. Phys. 302 (2015), p. 548-566.

[8] E. Johnsen and T. Colonius: J. Comput. Phys. 219 (2006), p. 715-732.

[9] E. Johnsen: Ph.D. thesis, California Institute of Technology (2007).

[10] K. W. Thompson: J. Comput. Phys. 68 (1987), p. 1-24.

[11] T. Kondo: Ph.D. thesis, Keio University (2019).

[12] T. Kondo and K. Ando: Phys. Fluids. 31 (2019), p. 011301. 